\section{Qualidade de vida na terceira idade: um conceito subjetivo}

\section{Quality of life in the elderly: a subjective concept}

\author{
Roberta Dalla Vecchia*1 \\ Tania Ruiz ${ }^{2}$ \\ Silvia Cristina Mangini Bocchi ${ }^{3}$ \\ José Eduardo Corrente ${ }^{4}$ \\ 'Estudante de Medicina, Faculdade de Medicina de Botucatu (FMB) - UNESP, \\ Bolsista de Iniciação Científica \\ ${ }^{2}$ Departamento de Saúde Pública, FMB-UNESP \\ ${ }^{3}$ Departamento de Enfermagem, FMB-UNESP \\ ${ }^{4}$ Departamento de Bioestatística, Instituto de Biociências (IB) - UNESP \\ campus de Botucatu
}

Estudo financiado pela FAPESP (bolsa de iniciação científica) - Processo 02/10077-6

*Correspondência: Distrito de Rubião Júnior s/n, 18618-970 Botucatu, SP - Brasil. E-mail:

robertabarra@uol.com.br

\section{Resumo}

Tendo-se em conta a expectativa de vida cada vez mais alta, vários estudos têm sido desenvolvidos de modo a contribuir para a melhoria da qualidade de vida na terceira idade. Objetivou-se conhecer a opinião dos idosos de um município de porte médio do interior paulista sobre o que é qualidade de vida, através de uma pergunta aberta que foi incluída como parte de um inquérito populacional sobre estilo e qualidade de vida. A análise da referida questão foi realizada através do método de Análise de Conteúdo. Posteriormente calcularam-se as freqüências das categorias obtidas, agrupadas segundo o método de Ward, e em seguida os idosos foram agrupados segundo o método de k-médias. Os resultados indicaram a existência de três grupos de idosos segundo sua definição de qualidade de vida: o primeiro valorizou a questão afetiva e a família; o segundo priorizou a obtenção do prazer e conforto; o terceiro poderia ser sintetizado como o idoso que identifica a qualidade de vida colocando em prática o seu ideário de vida. Sugere-se que, na implementação de ações, se objetive melhorar a qualidade de vida do idoso, levando-se em conta as magnitudes e as diferenças de cada grupo.

Palavras-chave: Idosos. Terceira idade. Estilo de vida. Qualidade de vida. 


\section{Abstract}

Considering that life expectancy has become increasingly higher, various studies have been conducted in order to improve the quality of life of the elderly. This study aimed to learn the opinions of the elderly living in a medium-size city in the state of São Paulo, Brazil with regard to the meaning of quality of life, by using an open question which was included as part of a population survey concerning lifestyle and quality of life. Such question was analyzed by means of the Content Analysis Method, and the frequencies of the categories obtained were later calculated. Frequencies were grouped according to the Method of Ward and the elderly were then grouped according to the k-means method. Results showed the existence of three groups according to the definition of quality of life: the first valued affection and family; the second prioritized leading a pleasant life based on the concepts of modernity; and the third could be summarized as consisting of elderly individuals who identified quality of life as putting into practice their life ideals. It is suggested that actions aimed at improving the quality of life of the elderly be implemented by taking into account the magnitudes and differences of each group.

Key Words: Elderly. Aging. Aged. Lifestyle. Quality of life.

\section{Introdução}

Diante da realidade inquestionável das transformações demográficas iniciadas no último século e que nos fazem observar uma população cada vez mais envelhecida, evidencia-se a importância de garantir aos idosos não só uma sobrevida maior, mas também uma boa qualidade de vida ${ }^{1}$.

O conceito de qualidade de vida está relacionado à auto-estima e ao bem-estar pessoal e abrange uma série de aspectos como a capacidade funcional, o nível socioeconômico, o estado emocional, a interação social, a atividade intelectual, o autocuidado, o suporte familiar, o próprio estado de saúde, os valores culturais, éticos e a religiosidade $^{2}$, o estilo de vida, a satisfação com o emprego e/ou com atividades diárias e o ambiente em que se vive ${ }^{3-5}$. O conceito de qualidade de vida, portanto, varia de autor para autor e, além disso, é um conceito subjetivo dependente do nível sociocultural, da faixa etária e das aspirações pessoais do indivíduo.

A literatura reflete (sic) que os instrumentos utilizados para análise da qualidade de vida de um modo geral não se adaptam aos idosos, seja porque têm uma abordagem unidimensional ${ }^{6}$ ou porque os idosos que se autodenominaram com boa qualidade de vida não a teriam segundo a interpretação dos instrumentos mencionados ${ }^{7}$. Parece, portanto, que existem aspectos característicos e multidimensionais ${ }^{5}$ que definem a qualidade de vida na faixa etária idosa.

Tendo em vista a variabilidade do conceito de qualidade de vida e sua subjetividade, com o propósito de se orientar as políticas para um envelhecimento bem sucedido, parece imprescindível conhecer o que, para a maioria dos idosos, está relacionado ao bem estar, à felicidade, à realização pessoal, enfim, à qualidade de vida nessa faixa etária.

Assim sendo, o objetivo deste estudo foi conhecer a opinião da população com 60 anos ou mais de um município de porte médio do interior do Estado de São Paulo sobre o que é, para eles, qualidade de vida. 


\section{Material e Métodos}

Foram estudados os significados de qualidade de vida segundo as respostas obtidas dos idosos, mediante a pergunta: "O que é qualidade de vida para o(a) $\mathrm{Sr}(\mathrm{a})$ ?”. Esta questão aberta foi inserida em extenso instrumento multidimensional utilizado em um inquérito conduzido no município de Botucatu no ano de 2003 e que teve como objeto de estudo a satisfação com a vida e o estilo de vida dos idosos. A pergunta foi feita ao idoso no final da aplicação do questionário, quando já havia certa aproximação com o entrevistador. Não foi induzido nenhum tipo de resposta. O entrevistador anotou em espaço de dez linhas deixado para esse fim o que foi falado pelo entrevistado. Trata-se de uma pesquisa realizada dentro de preceitos éticos, após parecer do Comitê de Ética em Pesquisa da Faculdade de Medicina de Botucatu e obtenção do termo de consentimento formal pósinformação dos idosos ou de representantes, devidamente habilitados para tal.

Para a composição do grupo estudado, inicialmente realizou-se uma amostra aleatória sistemática de domicílios residenciais de Botucatu, contemplando um quarto das residências de cada setor censitário urbano da cidade. Todos os moradores dos domicílios amostrados foram catalogados. Em uma segunda etapa, a partir do cadastro construído, amostrou-se aleatoriamente, dentro de cada setor censitário, residentes com idade superior a 60 anos. Suas residências foram visitadas por pesquisadores de campo treinados. Aqueles que se encontravam em casa em até quatro visitas, e que consentiram em participar do estudo, fizeram parte do grupo estudado.

Especificando-se um erro amostral $d$ igual a 0,05 , um valor $z$ igual a 1,96 relativo a um intervalo de confiança de $95 \%$ de confiança ( $a$ bilateral de 0,025); uma prevalência da característica de interesse $p$ igual a 0,5 e, desprezando-se o fator de correção da redução de heterogeneidade associada ao desenho de conglomerado $e$, o tamanho amostral mínimo necessário foi estimado, conforme a expressão abaixo ${ }^{8}$ :

$$
n \geq \frac{z_{\alpha / 2}^{2} p(1-p)}{d^{2}}
$$

fornecendo um total de 384 indivíduos. Destes, 4 não tinham 60 anos, 10 o endereço não foi localizado, 5 encontrou-se o domicílio fechado em mais de três visitas, 1 estava internado, 1 estava internado em São Paulo, 11 tinham ido a óbito e 3 eram casas de veraneio. Assim, foi feita a reposição de 16 casos: os domicílios fechados e os que tinham ido a óbito, totalizando 365 indivíduos. Foram perdidos, portanto, apenas $5 \%$ da amostra inicialmente estimada.

A análise das respostas da questão aberta constituiu-se de três fases: 1 - pré-análise, 2 exploração do material e 3 - tratamento dos resultados, inferências e a interpretação. $\mathrm{Na}$ pré-análise realizou-se a leitura de todas respostas e a transcrição das mesmas, visando formar o corpus de análise. A seguir, na fase 2, demarcamos as unidades de significação, também chamadas de unidades de registro, que são seguimentos do conteúdo, considerados como unidade de base, para a categorização e a contagem de freqüências ${ }^{9}$.

Na fase 3, as categorias identificadas na análise de conteúdo foram transformadas em variáveis binárias do tipo "sim" e "não" e, em seguida, foram calculadas as freqüências simples de cada variável.

Observando a freqüência com que cada categoria aparecia como afirmativa para cada idoso na definição de qualidade de vida, o método de Ward foi aplicado com a finalidade de agrupar a coincidência das respostas. Finalmente, aplicou-se o método das kmédias para avaliar a magnitude de cada agrupamento no conjunto dos idosos. Todas as análises foram feitas utilizando-se o programa STATISTICA v.5.0.

\section{Resultados}

Da análise das respostas dos 365 idosos estudados acerca da sua compreensão sobre qualidade de vida emergiram onze categorias com seus significados, que serão apresentadas a seguir em ordem decrescente quanto às ocorrências nos relatos dos indivíduos: 
Categoria 1 - Preservando os relacionamentos interpessoais (49\% - 179 idosos): significa poder manter fortalecidos em número e qualidade os vínculos com a família, contribuindo se possível com a educação de filhos e netos, bem como estendendo a vizinhos e amigos, solidificando sua rede de suporte social na senectude. As unidades de registro que retratam a categoria foram agrupadas de maneira que, para o idoso, ter qualidade de vida é "ter um bom convívio social com todos, particularmente com vizinhos e amigos; um bom relacionamento familiar, incluindo uma boa criação e educação dos filhos e netos;capacidade para estabelecer contato com as pessoas e fazer novas amizades; e um bom relacionamento com o cônjuge."

Categoria 2 - Mantendo uma boa saúde (38,9\% - 142 idosos): destes, $29 \%$ (41 idosos) relativos especificamente aos hábitos saudáveis. Para eles, qualidade de vida é poder adotar hábitos de vida considerados saudáveis, relativos à alimentação, ao sono, à prática de exercícios físicos regulares e ao não uso de drogas. "Ter uma alimentação balanceada; dormir adequadamente; praticar esportes; e não usar droga são indispensáveis para a manutenção da qualidade de vida."

Categoria 3 - Mantendo o equilíbrio emocional (34,25\% - 125 idosos): promover qualidade de vida é dispor de tranqüilidade, bom humor e sentir-se satisfeito com a vida, atributos relativos a saúde mental que ajudam os idosos a se manterem fortalecidos no enfrentamento das atividades diárias e dos desafios impostos pela vivência. As unidades de registro que compõem a categoria foram agrupadas de tal maneira que, para o idoso, ter qualidade de vida é relatado como "ter motivação para as atividades diárias e para novas atividades elou desafios; tranqüilidade; satisfação com a vida; bom humor e estabilidade emocional, mesmo em situações desfavoráveis."

Categoria 4 - Acumulando bens materiais (28,5\% - 104 idosos): significa que, ao longo da vida, a pessoa precisa ir conquistando e reunindo bens que possam contribuir para a sua segurança e conforto na senectude. Para tanto, acham necessário ter um bom salário, sabendo poupá-lo, bem como dispor de casa própria. São condições que poderão lhes conferir autonomia quanto: à alimentação, vestuário, transporte, obtenção de eletrodomésticos, assistência médica e medicamentos necessários. As unidades de registros que retratam a categoria foram agrupadas e expressas de tal maneira que, "conquistar a vida com qualidade é ter um bom salário; saber utilizar o dinheiro sem fazer dívidas; poder comprar alimentos, vestuário, eletrodomésticos e automóveis; ter conforto; ter casa própria; poder consultar médicos particulares e comprar os remédios necessários."

Categoria 5 - Tendo lazer $(22,46 \%$ - 82 idosos): significa entretenimento, conjunto de atos que promovem divertimento, distração e relaxamento, contribuindo para a qualidade de vida dos idosos, tais como: "viajar, passear, andar de bicicleta, ouvirmúsica, dançar, plantar, pescar, fazer churrasco, encontrar amigos, jogar baralho, cuidar de animais de estimação e fazer algum tipo de artesanato como bordado, tricô ou crochê."

Categoria 6 - Trabalhando com prazer $(6,3 \%$ - 23 idosos): significa que o trabalho, principalmente quando desempenhado com prazer, é um meio para se obter qualidade de vida.

Categoria 7 - Vivenciando a espiritualidade $(8,22 \%$ - 30 idosos): significa a adesão a um conjunto de dogmas e doutrinas que constituem a anuência pessoal aos desígnios e manifestações relativas à religião e, portanto, para os entrevistados, "qualidade de vida é, também, ter religião e fé."

Categoria 8 - Praticando a retidão e a caridade $(4,93 \%$ - 18 idosos): significa a sua satisfação em poder praticar a solidariedade e a honestidade. As unidades de registros que representam a categoria foram agru- 
padas de tal maneira que qualidade de vida para o idoso é, também, poder "praticar o bem junto ao seu semelhante e saber que é honesto, não prejudicando, mas ajudando o semelhante, e cumprir adequadamente suas obrigações."

Categoria 9 - Acessando o conhecimento $(4,11 \%$ - 15 idosos): qualidade de vida significa ter acesso à educação, apropriando-se de conhecimentos no decorrer da vida, por meio de estudos eleituras. As unidades de significação que compreendem a categoria acessar conhecimento como componente da qualidade de vida são: "poder ler e estudar ou mesmo ter estudado no passado."

Categoria 10 - Vivendo em ambiente favorável (2,46\% - 9 idosos): qualidade de vida significa poder morar em locais tranqüilos, onde ainda se consegue preservar a segurança e a despoluição ambiental. Para eles, ter qualidade de vida é "poder viver em locais seguros e despoluidos, como se preserva ainda no campo, viver em um ambiente não poluído e sem violência"

\section{Categoria 11 - Não respondendo}

(3,56\% - 13 idosos): significa aqueles idosos que preferiram omitir significados à qualidade de vida, tais como: "eu não saberia responder".

Um dos idosos entrevistados, afirmou que qualidade de vida é "uma condição variável de pessoa para pessoa".

De posse das categorias, a análise de agrupamentos segundo o método de Ward, que analisa a coincidência das categorias nas respostas dos idosos acerca do significado de qualidade de vida, permitiu-nos chegar à obtenção de três grupos de respostas, que estão representados na Figura 1.

No primeiro grupo, observa-se que as variáveis relacionamentos interpessoais, equilíbrio emocional e boa saúde foram as que mais apareceram juntas nas respostas individuais.

No segundo grupo, por sua vez, as variáveis mais coincidentes foram: hábitos saudáveis, lazer e bens materiais .

O terceiro foi constituído pelas situações nas quais a qualidade de vida estava associada à espiritualidade, ao trabalho, à retidãoe à caridade, ao conhecimento e aos ambientes favoráveis.

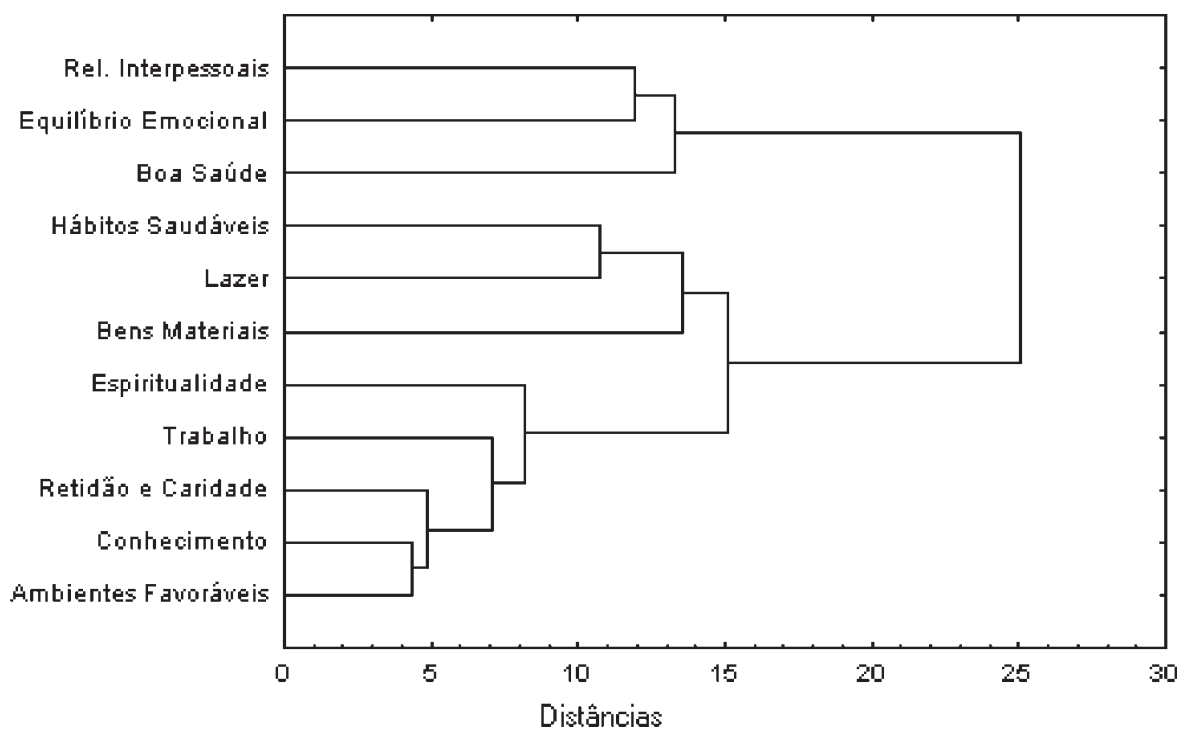

Figura 1. Dendrograma obtido pelo Método de Ward para a avaliação das respostas sobre qualidade de vida por idosos da cidade de Botucatu - SP.

Chart 1. Dendogram obtained by the Ward's Method to evaluate the answers of Botucatu's elderly about quality of life. 
O método das k-médias, utilizado para quantificar os idosos com respostas coincidentes, continuou identificando três grupos, dessa vez de idosos. As respostas para cada grupo podem sugerir interpretações sobre o que é qualidade de vida da seguinte maneira:

Grupo 1 - Qualidade de vida significa dispor de uma rede social de suporte sólida, associada à saúde física e mental. Este grupo agrega as categorias: preservando os relacionamentos interpessoais (49\%), mantendo uma boa saúde $(38,9 \%)$, mantendo o equilíbrio emocional $(34,25 \%)$.

Grupo 2 - Qualidade de vida significa servir-se de autonomia financeira conquistada durante a vida, para assegurar recursos para a senectude, associada às práticas de hábitos saudáveis e de entretenimento. Este grupo reuniu as categorias: mantendo uma boa saúde (38,9\%), acumulando bens materiais $(28,5 \%)$, tendo lazer $(22,46 \%)$.

Grupo 3 - Qualidade de vida significa poder viver em local seguro e despoluído, associado ao acesso a conhecimentos ao longo da vida, ao prazer no trabalho e a práticas de espiritualidade, honestidade e solidariedade. Este grupo reuniu as categorias: vivenciando a espiritualidade (8,22\%), trabalhando com prazer $(6,3 \%)$, praticando a retidão e a caridade $(4,93 \%)$, acessando o conhecimento $(4,11 \%)$ e vivendo em ambiente favorável (2,46\%).

No grupo em que foram valorizados os relacionamentos interpessoais, equilíbrio emocional e boa saúde encontramos 132 idosos $(36,2 \%)$. No grupo em que foram valorizados hábitos saudáveis, lazer e bens materiais, encontramos 145 idosos (39,7\%) e no grupo em que foram valorizados espiritualidade, trabalho, retidão e caridade, conhecimento e ambientes favoráveis encontramos 88 idosos $(24,1 \%)$.

\section{Discussão}

Apesar da variabilidade individual do conceito de qualidade de vida, conseguiu-se no presente estudo, generalizar alguns aspectos considerados importantes pelos ido- sos da cidade de Botucatu-SP para a conquista de uma vida feliz.

Os achados coincidiram com os de outras populações idosas e, além disso, houve semelhanças entre aquilo que os entrevistados consideraram importante para a obtenção da vida com qualidade e questões abordadas por instrumentos que se destinam à quantificação de qualidade de vida ${ }^{10-13}$. Pesquisas qualitativas ${ }^{1,4,5}$ sobre o conceito de qualidade de vida encontraram como resultados a valorização pelos idosos do bom relacionamento com a família, com amigos e da participação em organizações sociais; da saúde; de hábitos saudáveis; de se possuir bem-estar, alegria e amor; de uma condição financeira estável; do trabalho; da espiritualidade; de se praticar trabalhos voluntários e de se poder aprender mais. As citações da literatura confirmam as categorias encontradas neste estudo. Os agrupamentos formados através da coincidência das respostas, sugerem perfis de idosos de acordo com a afinidade obtida entre as categorias.

\section{Conclusão}

Foram identificados três perfis de idosos no município de Botucatu - SP, segundo a definição que eles deram sobre o que era qualidade de vida: o primeiro mencionou situações referentes a relacionamentos interpessoais, equilíbrio emocional e boa saúde, ou seja, é o idoso que prioriza a questão afetiva e a família; o segundo grupo mencionou hábitos saudáveis, lazer e bens materiais, ou seja, é o idoso que prioriza o prazer e o conforto; e o terceiro grupo, que mencionou espiritualidade, trabalho, retidão e caridade, conhecimento e ambientes favoráveis, poderia ser sintetizado como o idoso que identifica como qualidade de vida conseguir colocar em prática o seu ideário de vida.

Em vista dos resultados obtidos, sugerese que, na implementação de ações que objetivem melhorar a qualidade de vida do idoso, sejam consideradas as magnitudes e as diferenças de cada grupo sobre o que eles mesmos valorizam na busca do bem-estar na terceira idade. 


\section{Agradecimentos}

Dr Ricardo Carlos Cordeiro, Prof. LivreDocente do Departamento de Saúde Públi- ca, FMB, UNESP, por haver disponibilizado seus dados com as famílias do município de Botucatu já amostradas e pelas sugestões ao artigo.

\section{Referências}

1. Fleck MPA, Chachamovich E, Trentini CM. WHOQOLOLD Project method and focus group results in Brazil. Rev Saúde Publica 2003; 37(6): 793-9.

2. Santos SR, Santos IBC, Fernandes MGM, Henriques MERM. Elderly quality of life in the community: application of the Flanagan's Scale. Rev Latino Am Enfermagem 2002; 10(6): 757-64.

3. Velarde JE, Avila FC. Methods for quality of life assessment. Salud Pública Méx 2002; 44(4): 349-61.

4. Isang EYL, Liamputtong P, Pierson J. The views of order chinese people in Melbourne about their quality of life. Ageing \& Society 2004; 51-74.

5. Bowling A, Gabriel Z, Dakes J, Dowding LM, Evans O, Fleissig A et al. Let's ask them: a national survey of definitions of quality of life and its enhancement among people aged 65 and over. Int J Aging Hum Dev 2003; 56(4): 269-306.

6. Higgs P, Hyde M, Wiggins R, Blane D. Researching quality of life in early old age: the importance of the sociological dimension. Soc Policy Adm 2003, 37(3): 239-52.

7. Strawbridge WZ, Wallhagen MI, Cohen RD. Successful aging and well-being: self-rated compared with Rowe and Kahn. Gerontologist 2002, 42(6): 727-33.
8. Kish L. Muestreo de encuestas. Mexico: Editorial Trillas; 1972.

9. Bardin L. Análise de conteúdo. Lisboa: Persona; 1977.

10. Xavier FMF, Ferraz MPT, Marc N et al. A definição dos idosos de qualidade de vida. Rev Bras Psiquiatr 2003; 25(1): 31-9.

11. Assantachai P, Maranetra N. Nationwide survey of the health status and quality of life of elderly Thais attending clubs for the elderly. J Med Assoc Thai 2003; 86(10): 938-46.

12. Baiyewu O, Jegede RO. Life satisfaction in elderly Nigerians: reliability and factor composition of the life satisfaction Index Z. Age Ageing 1992; 21: 256-61.

13. Dello Buono M, Urciuoli O, De Leo D. Quality of life and longevity: a study of centenarians. Age Ageing 1998; 27: 207-16.

recebido em: 14/10/04 versão final reapresentada em: 23/05/05 aprovado em: 09/06/05 